\title{
The Wave Normals of Magnetospheric Chorus Emissions Observed on Board GEOS 2
}

\author{
M. Hayakawa, ${ }^{1,2}$ Y. Yamanaka, ${ }^{1,3}$ M. Parrot, ${ }^{2}$ and F. Lefeuvre ${ }^{2}$
}

\begin{abstract}
The wave normal directions of magnetospheric chorus emissions have been analyzed by using data from the geostationary GEOS 2 satellite which is located in the equatorial region at $L=6.6$. As the result of analyses for a specific day of December 19,1979, chorus is found to be generated in conjunction with substorms and at local times from around midnight to afternoon. Two types of chorus are observed: impulsive burstlike and rising tone. The chorus during midnight to late morning hours is impulsive, and at local times from noon to dusk we notice an increase in the occurrence of rising tone chorus. The chorus spectra have shown the importance of the characteristic frequency of $f_{H} / 2\left(f_{H}\right.$ is the electron gyrofrequency). We notice the impulsive chorus only in the lower band $\left(f<f_{H} / 2 ; f\right.$ is the emission frequency) at local times from $\sim 0300$ to $\sim 0700$, and then at LT $=\sim 0800$ to $\sim 1600$ the predominant frequency of the emissions, whether impulsive or rising tone, lies in the upper band $\left(f>f_{B}\right)$ 2) with infrequent occurrence in the lower band, and there is obviously no correlation between the elements in both bands. However, there are several intense tones which seem to be correlated in both bands for the strong two-banded impulsive chorus emissions observed only at $\mathrm{LT} \simeq 0800$. This correlation might be related with a notch in chorus risers at $f \simeq f_{H} / 2$ caused by Landau damping, as previously suggested. The wave normals are determined by using only the magnetic field data. The direction finding studies have been made for impulsive and rising tone chorus, separately, which have yielded different results. The lower band is discussed first. The wave normals of the rising tones having values of $d f / d t$ as normally observed are found to take very small angles $\left(5^{\circ}-20^{\circ}\right)$. Then it is found that the rising tones with increased values of $d f / d t$ have shown larger wave normal angles of $30^{\circ}-45^{\circ}$. These relatively small wave normal angles for the lower band give further support to the loss cone instability as the generation mechanism. The impulsive chorus is found to take slightly larger wave normal angles (about $5^{\circ}$ ) than most rising tones. Further, it may be suggested that different types of chorus are associated with different wave normals. Next we are concerned with the upper band chorus, whose generation mechanism is not well agreed upon. It is shown that the impulsive chorus in the upper band is generated with its wave normal close to the oblique resonance cone. This suggests that the upper band chorus is quasi-electrostatic, and it is furthermore confirmed by the estimated ratio, $c|B| /|E|(|E|$ and $|B|$ are the total electric and magnetic field intensities; $c$ is the light velocity). The rising tones in the upper band, however, have indicated a difference such that the closeness of their wave normals to the oblique resonance cone is much less distinct. An intensive comparison is made between the direction finding results for the upper band and the theoretical mechanisms so far proposed in order to elucidate the possible generation mechanism of the upper band chorus.
\end{abstract}

\section{INTRODUCTION}

Morphological features of chorus emissions throughout the magnetosphere have been investigated by several earlier workers, including Oliven and Gurnett [1968] and Taylor and Gurnett [1968] based on the INJUN satellites, Dunckel and Helliwell [1969] based on the OGO 1 VLF measurements, and Russell et al. [1969] and Russell and Holzer [1970] using the OGO 3 data. These studies have revealed that chorus is observed mainly at $L$ values between the plasmapause and magnetopause and at all local times (LT), but predominantly in the dayside magnetosphere.

The midnight sector has recently been considered to be an especially interesting region for chorus because chorus is generated during magnetospheric substorms and storms [Tsurutani and Smith, 1974, 1977; Thorne et al., 1974, 1977; Hayakawa et al., 1977] by freshly injected electrons [Anderson and Maeda, 1977; Tsurutani and Smith, 1977; Thorne et al., 1977; Tsurutani et al., 1970; Isenberg et al., 1982]. The emissions are further found to be localized to the magnetic equator [Russell et al., 1969; Burtis and Helliwell, 1969; Tsurutani and Smith,

\footnotetext{
${ }^{1}$ Research Institute of Atmospherics, Nagoya University.

${ }^{2}$ Laboratoire de Physique et Chimie de l'Environnement, Centre National de la Recherche Scientifique.

${ }^{3}$ Now at Radio Research Laboratories, Tokyo.

Copyright 1984 by the American Geophysical Union.

Paper number 4A0058.

0148-0227/84/004A-0058\$05.00
}

$1974,1977]$, the region where the wave growth rate is expected to be largest.

An interesting property of the frequency spectrum of chorus has been found by Tsurutani and Smith [1974], who have shown that chorus is composed of two bands of frequencies: one above one-half the electron gyrofrequency $\left(f_{H} / 2\right)$ (the upper band) and the other below $f_{H} / 2$ (the lower band). This characteristic two-band structure has been found to be very persistent for chorus and has been experimentally investigated in detail by Maeda et al. [1976] and Anderson and Maeda [1977].

The lower band chorus has recently been interpreted as being generated by substorm electrons with an anisotropic loss cone distribution, and the theoretical aspects are well explained by the theory of Kennel and Petschek [1966] [Burton and Holzer, 1974; Anderson and Maeda, 1977; Hayakawa et al., 1977; Tsurutani et al., 1979; Isenberg et al., 1982]. On the other hand, the generation mechanism of the chorus in the upper band is not well agreed upon. When the two-band structure was originally discovered, Tsurutani and Smith [1974] ascribed the $f_{H} / 2$ gap to the damping mechanism because chorus tones which cut across the $f_{H} / 2$ gap could clearly be observed. Maeda et al. [1976] have found that there is no coherence between the upper and the lower band chorus, and they have postulated that the upper band is locally generated whistler mode waves near the satellite and the lower band is whistler mode waves generated in regions of lower magnetic field farther out in the magnetosphere, followed by the propagation to the satellite, yielding a cutoff at $f_{H} / 2$. Curtis [1978] 

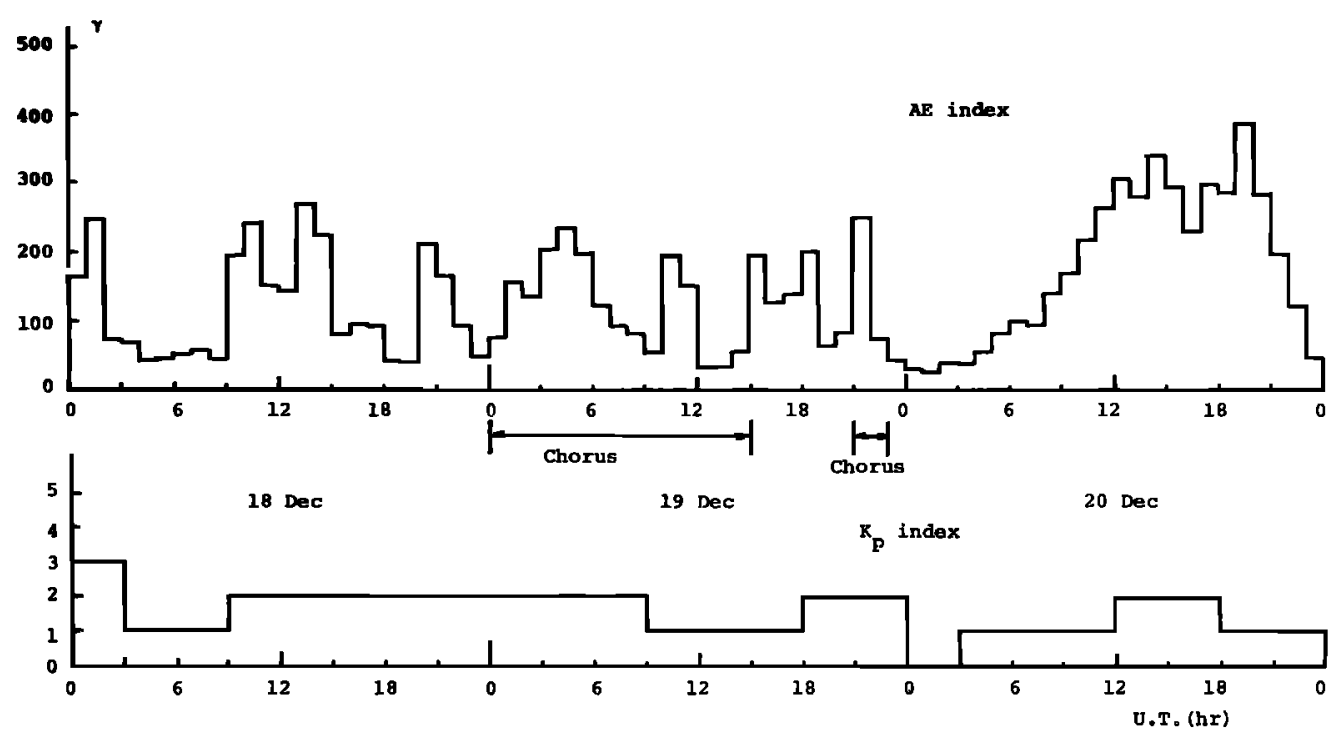

Fig. 1. Temporal variation of geomagnetic activity ( $A E$ and $K p$ indices) during a few days around December $19,1979$. The time interval when chorus was detected is also indicated.

has presented an interpretation that the upper and lower bands in the case of $f_{p}$ (electron plasma frequency) less than $f_{H}$ are both generated locally, the lower being represented by whistler mode and the upper band being described by ordinary mode. Burtis and Helliwell [1976] have shown by detailed ray tracing studies the ability of propagation effects to possibly produce a bimodal distribution of chorus emissions.

Information concerning the distribution of wave normal vectors of chorus is considered to be a valuable tool, not only in providing further evidence for the existing generation theory but also in suggesting new generation mechanisms. Wave normals of chorus mainly in the off-equatorial region in the outer magnetosphere have been extensively studied by Burton and Holzer [1974], but the analyses of wave normal directions just at the equatorial plane, the likely source region, such as were done by Cornilleau-Wehrlin et al. [1976], are important to obtain direct evidence of the generation mechanism.

In the present paper, wave normal directions of magnetospheric chorus observed at the magnetic equator have been determined with data from the geosynchronous GEOS 2 satellite located at $L=6.6$. The analyses have been carried out for both the upper and the lower band chorus by means of our newly developed method in which we apply the maximum likelihood estimation to the observed spectral matrices. Section 2 deals with the data base used for the direction findings, and we present the method of our direction finding to determine the wave normal directions. Then in section 3 we describe the characteristics of the observed emissions. Section 4 is concerned with the final results of direction findings of chorus in both bands. Finally, we discuss the generation mechanisms of both band chorus in section 5 .

\section{Data Base and the Direction Finding TEChNIQUe}

For the detailed study of electromagnetic waves below the electron gyrofrequency $f_{H}$, several sensor combinations and different modes of on-board data processing have been used. For the detailed description of the GEOS S-300 experiment, see Jones [1978] or $S-300$ experimenters [1979]. Here, the data we are treating have been collected by the so-called S-300 "survey mode" (6 min of identical operations in passive and active modes every hour) on the geosynchronous satellite GEOS 2, which is located in the equatorial region at $L=6.6$. Since the electric field components which are measured by small electric antennas [S-300 experimenters, 1979] are not very sensitive and, above all, are strongly perturbed over one half of each 6-s spin cycle [Wrenn, 1978], we only use the data from three magnetic sensors. The signals are subjected to two on-board analyses: the step frequency analyzers (SFA's) and the correlator. The SFA's, which may be connected to any sensor combination, have a bandwidth of $300 \mathrm{~Hz}$ and are swept in frequency in the range $0-77 \mathrm{kHz}$. Before being telemetered to the ground, the signals are transposed in frequency, passed through identical low-pass filters at $450 \mathrm{~Hz}$,
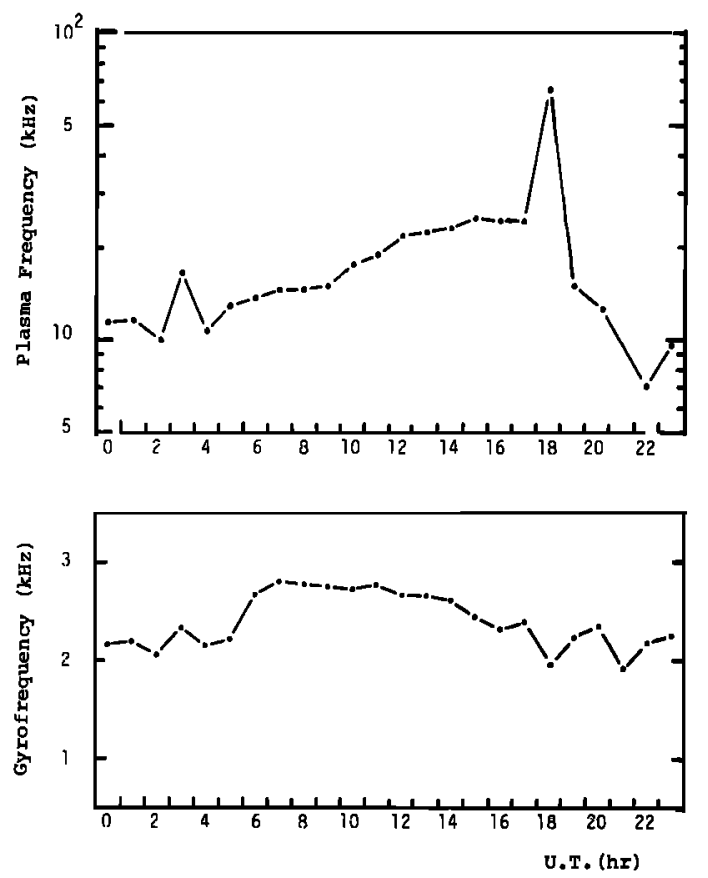

Fig. 2. Temporal variation of the electron plasma frequency $f_{p}$ and electron gyrofrequency $f_{H}$ on December 19, 1979. 


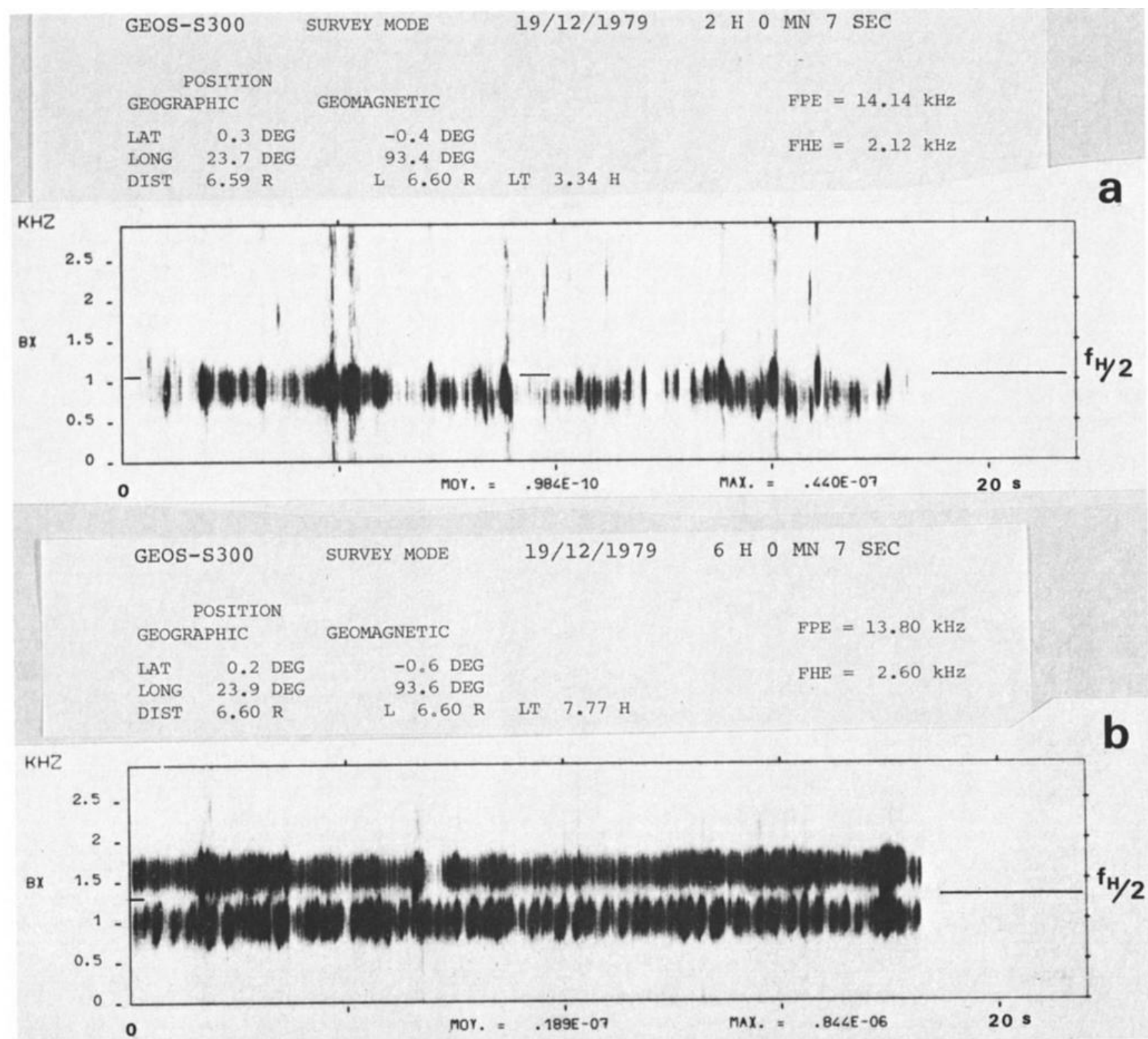

Fig. 3. Two examples of impulsive (or burstlike) chorus. (a) UT $=0200$ (LT $=0320: 24$ ). The chorus in the lower band with its uppermost frequency sometimes extending above $f_{B} / 2$. (b) UT $=0600$ (LT $=0746: 12$ ). The two-banded chorus emissions with a conspicuous missing frequency at $f_{B} / 2$; the chorus emissions in both bands appear to be not correlated with each other.

and sampled at $1.488 \mathrm{kHz}$. The correlator output, after suitable Fourier transformation on the ground, yields a spectral resolution of $2,5 \mathrm{kHz}$ (ELF part of the survey mode) or 5.0 $\mathbf{k H z}$ (VLF part). The direction findings are performed from the magnetic SFA data in the VLF part. For the sake of a better visualization, high-resolution spectrograms $(2.5 \mathrm{kHz})$ taken during the ELF part are represented here, with the consequence that they do not exactly coincide with the SFA data. In any case the VLF part follows the ELF one, and the time shift is always less than $22 \mathrm{~s}$. A few seconds after the end of the VLF part, the plasma frequency is estimated successively by two active experiments: S-301 and S-304 [S-300 experimenters, 1979; Decreau et al., 1978]. The two sets of plasma frequencies are used either as a cross-check or as an alternative when one experiment fails.

After looking at many survey mode data from the GEOS 2 satellite, we selected one specific day, December 19, 1979, for further detailed direction finding analyses, because we had recorded a lot of chorus on this day. Some of the spectrograms will be presented in the next section.

Wave normal directions are estimated from the SFA data, using the method developed by Buchalet and Lefeuvre [1981]. First, the signals associated with the magnetic components are Fourier-transformed. Then, at each frequency we are concerned with, we estimate a $3 \times 3$ spectral matrix consisting of the mean autopower spectrum of each of the three components and the mean cross-power spectra between two of the three components. Before carrying out the direction finding, we have to determine the model of propagation (one- or twodirection model) by means of the three eigenvalues, $\lambda_{1}, \lambda_{2}$, and $\lambda_{3}$ (ranked in decreasing magnitude), of the observed spectral matrix at each frequency. The criterion in distinguishing between the one- and two-direction model is rather arbitrary; we use, on some occasions, a combination of the two ratios $\alpha=$ 


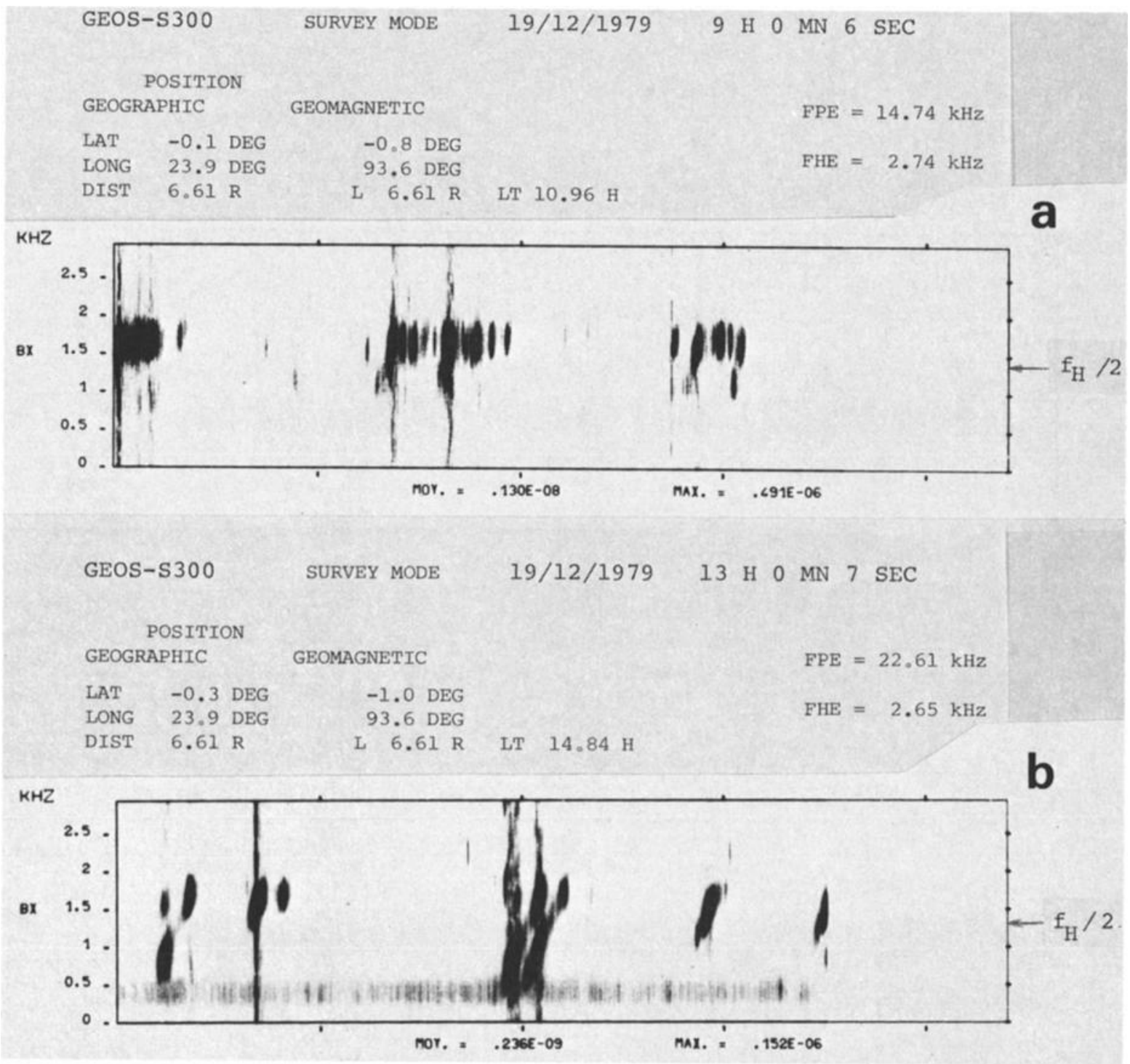

Fig. 4. (a) $\mathrm{UT}=\mathbf{0 9 0 0}(\mathrm{LT}=1057: 36)$. Rising tone chorus emissions are seen in the upper band, being simultaneous to the impulsive chorus; $d j / d t \simeq 5.4 \mathrm{kHz} / \mathrm{s}$. (b) $\mathrm{UT}=1300$ ( $(\mathrm{LT}=1450: 24)$. Several examples of rising tone chorus are detected not only in the upper band, but also in the lower band; $d f / d t \simeq 1.3-1.9 \mathrm{kHz} / \mathrm{s}$.

$\lambda_{2} / \lambda_{1}$ and $\beta=\lambda_{3} / \lambda_{2}$, the thresholds for which are chosen depending on the degree of confidence required [Buchalet and Lefeuvre, 1981; Lefeuvre et al., 1982], and, in other cases, the degree of polarization; $\lambda_{1} /\left(\lambda_{1}+\lambda_{2}+\lambda_{3}\right)>n(=0.90-0.95)$ is used [Lefeuvre et al., 1982]. In the present paper we adopt the former criterion such that if $\alpha<0.1$, the field is considered to be a single plane wave, and if $\alpha>0.1$ and $\beta<0.4$, we assume the two-plane wave model. After determining the propagation model, the maximum likelihood concept is made use of to deduce the wave normal directions of emissions [Buchalet and Lefeuvre, 1981]. One more thing we have to mention here is the assumption that the propagation of waves is described by the cold plasma theory, which will be important in interpreting the results in sections 4 and 5 .

In what follows, we adopt a Cartesian coordinate system $O_{x y z}$ where the $z$ axis is parallel to the earth's magnetic field $\mathbf{B}_{0}, O x$ is in the magnetic meridian plane containing the point of observation and the $x$ axis is directed away from the earth, while $O y$ completes the orthogonal set and the $y$ axis is eastward. The wave normal direction (k) is characterized by the polar angle $\theta$ between $\mathbf{k}$ and $\mathbf{B}_{0}$ and the azimuthal angle $\phi$, the origin of which is in the meridian plane.

\section{Characteristics of Observed Chorus}

One specific day, December 19, 1979, was selected, and Figure 1 illustrates the temporal variation of the geomagnetic activity ( $A E$ and $K p$ indices) during a period of a few days around that day. The time interval when chorus was recorded on December 19 is also indicated in the figure. Figure $1 \mathrm{dem}$ onstrates that a lot of successive substorms and their associated particle injections occur on December 18 and 19, and it is apparent that the occurrence of chorus is substorm related, as is already mentioned in the introduction.

Figure 2 shows the diurnal variation of the plasma parameters $f_{p}$ and $f_{H}$ measured by the satellite at the equator. The details of the measurements of $f_{p}$ and $f_{H}$ are mentioned by $S-300$ experimenters [1979], Decreau et al. [1978], and Etcheto and Bloch [1978]. As can be seen from the figure, $f_{p}$ is much larger than $f_{H}$ at all UT's. Then, since the emission frequency 
is always below $f_{H}$ and above the lower hybrid resonance frequency, there exists only one magnetoionic mode, i.e., the whistler mode.

The frequency-time characteristics of VLF emissions are considered to be related to nonlinear processes associated with the generation mechanism. Chorus is defined, in the present paper, as the signal containing discrete structures (or elements) and not necessarily risers. Two types of chorus are discussed in the present paper. The first type is an impulsive, burstlike chorus which appears as a vertical trace in the spectrum. Two examples of this type are shown in Figure 3. The other is the normal rising tone chorus whose spectral trace exhibits an increase in frequency with time. Two examples are given in Figure 4. Rising tone chorus is very popular and predominant and is reported by many workers cited in the introduction. Impulsive chorus, too, is not unpopular and unusual, because it is relatively often detected on board the GEOS satellites [Gibbons and Ward, 1981; Cornilleau-Wehrlin et al., 1978] and on OGO 5 [Cornilleau-Wehrlin et al., 1976]. Other types, such as falling tones and flat tones, are recorded as well by Tsurutani and Smith, [1977] and Cornilleau-Wehrlin et al. [1976]. Figure $5 a$ illustrates the temporal variation of the percentage occurrence of impulsive chorus, which is roughly estimated by counting the elements in the survey mode spectrograms such as are shown in Figures 3 and 4. Chorus has been detected in the UT intervals $0000-1400$ (the corresponding LT is 0115:36-1546:48) and 2100-2200 (LT = 2222:12-2319:48). During the LT interval in between, some VLF noises are observed, but we cannot identify them as chorus. Thus we tend to see chorus from around midnight to daytime, but no chorus between 1600 and $2100 \mathrm{LT}$. This distribution of chorus as a function of LT seems to be in good agreement with previous studies by Tsurutani and Smith [1974, 1977], Thorne et al. [1974, 1977], and Cornilleau-Wehrlin et al. [1978]. This LT dependence of chorus occurrence can be understood as follows [e.g., Tsurutani and Smith, 1977]. Substorm electrons are injected into the nightside magnetosphere at approximately local midnight, and because of the strong inward convection we expect an increased particle flux and pitch angle anisotropy, leading to the wave generation. Further, the chorus from 0700 to $0900 \mathrm{LT}$ is intensified (although this is not shown in the text), which can be explained by enhanced waveparticle interactions in this sector due to an increase in the ambient plasma density from the sunlit ionosphere.

At the top of Figure $5 a$ we have indicated more detailed information on the chorus band. When only the notation $l$ is designated, we have had the emissions only in the lower band, and the reverse situation is expressed by $u$. When the emissions take place predominantly in the upper band, with infrequent emissions in the lower band, the less predominant band is indicated in parentheses. The notation $(u)$ means the reverse situation. Figure $3 a$ illustrates a series of impulsive chorus elements only in the lower band with their uppermost frequency sometimes extending into the upper band, which are observed at UT $=0200$. When the two bands are simultaneously recorded, with less numerous occurrence in either of the two bands (although no good examples of these are illustrated in the text, one can find this phenomenon in Figure $4 b$ ), we have found that there is no correlation of the elements in both bands. Simultaneous occurrence of strong emissions in both bands is obtained only at UT $=0600$ ( $\mathrm{LT}=0746: 12$ ), and an example is shown in Figure $3 b$. A close inspection suggests that there are several intense tones which seem to be correlated in the upper and lower bands. As far as the corre-
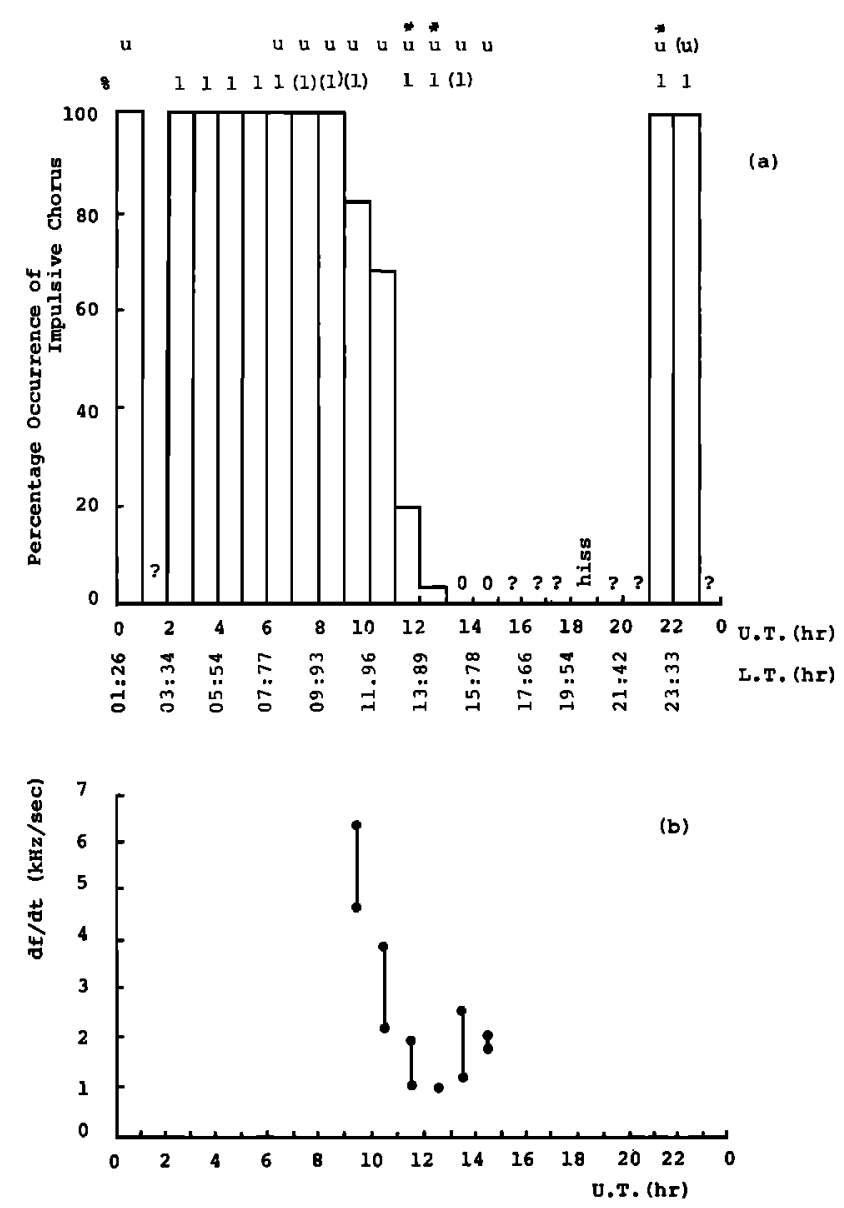

Fig. 5. (a) Temporal variation of the percentage occurrence of impulsive chorus, as estimated from the spectrograms of the survey mode. The zeros at two UT's indicate that no impulsive chorus has been recorded and that all of the observed chorus emissions are rising tones. The question marks for some UT's mean that the observed waves cannot be identified as chorus for certain. At the top we add the information on the chorus band; $u$ and $l$ mean the upper and the lower band, respectively. For UT $=0600$ we observe the simultaneous occurrence of strong emissions in both bands, the example being shown in Figure $3 b$. When $u$ and $l$ are simultaneously indicated, with either of the two in parentheses, the band in parentheses is less predominant, indicating the infrequent occurrence of emissions in that band. Then, for the UT's with asterisks, the emissions do not show any gap at $f_{H} / 2$, as illustrated in Figure 6. (b) Temporal variation of the rate of frequency increase with time, $d f / d t$, of rising tone chorus.

lation between the two bands is concerned, conflicting results have been presented so far. No correlation has been found by Maeda et al. [1976], but Tsurutani and Smith [1974] have presented examples in which both bands appear to be clearly connected except a strong extinction band at $f_{H} / 2$.

As the LT increases from 1100 to 1600 (UT $=0900-1400$ ), the percentage occurrence of rising tone chorus becomes enhanced, but we have to mention that the total chorus events become less numerous. The variation of the rate of frequency with time $(d f / d t)$ of the rising tone is summarized in Figure $5 b$. The $d f / d t$ decreases abruptly from $\mathrm{LT}=1057: 36$ to $1157: 36$, followed by a relatively constant value $(0.9-2.4 \mathrm{kHz} / \mathrm{s}$, but predominantly $\sim 1.5 \mathrm{kHz} / \mathrm{s}$ ) from $1353: 24$ to $1546: 48 \mathrm{LT}$. Burtis and Helliwell [1976] have estimated that the medium $d f / d t$ is $0.77 \mathrm{kHz} / \mathrm{s}$ at $L \sim 6$ and that it shows some decrease with increasing $L$ values. Thus even our smallest values of $d f / d t$ seem to be slightly larger than the value obtained by 


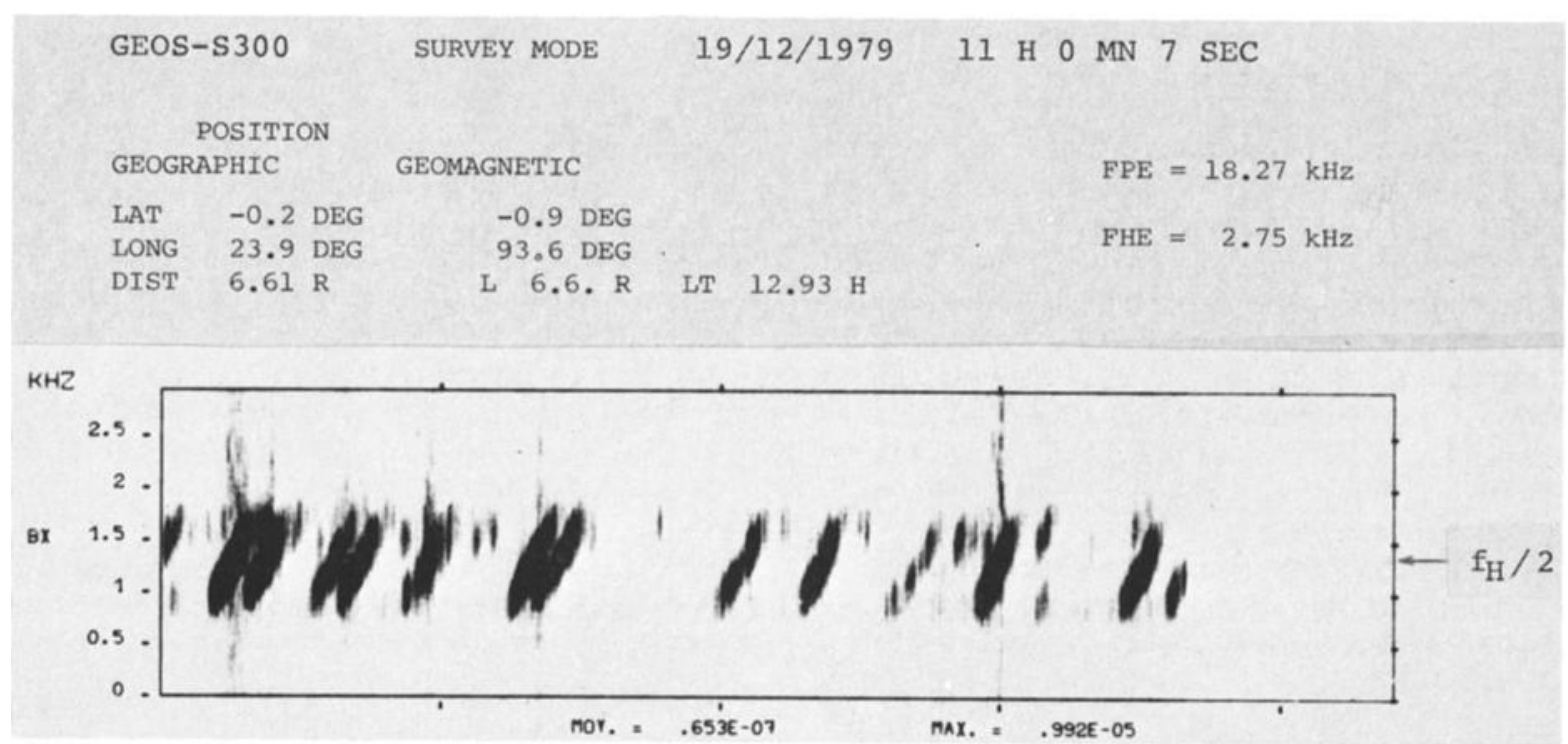

Fig. 6. Example of rising tone chorus which does not show any conspicuous change at $f_{H} / 2 . \mathrm{UT}=1100(\mathrm{LT}=1157: 36$, near noon); $d f / d t \simeq 1.3 \mathrm{kHz} / \mathrm{s}$. This kind of chorus is also recorded at $\mathrm{UT}=1200$ and 2100 .

them. In Figure $4 a$ a small number of rising tone chorus emissions in the upper band are detected that are simultaneous to a larger number of impulsive chorus emissions, and their $d f / d t$ is very large, i.e., $d f / d t=5.4 \mathrm{kHz} / \mathrm{s}$. Figure $4 b$ presents several rising tones mainly in the upper band, and also in the lower band. It does not appear that the rising tone elements in both bands are connected or correlated, just as in the case of many impulsive chorus elements in Figure $3 b$. The LT dependence of chorus structure in Figure $5 a$ is then compared with the previous study by Tsurutani and Smith [1977], who have stated that flat tones are detected in the evening-postmidnight sector, while falling tones are primarily observed in the dayside at low $L(5-6)$ and rising tones are also detected at $L$ less than 6.8 .

The examples in Figures 3 and 4 have clearly demonstrated the importance of the characteristic frequency $f_{H} / 2$ in the chorus whether it is impulsive or rising the, but some rising tone chorus was observed which exhibit, no conspicuous change at $f_{H} / 2$ (no gap at that frequency) at $\mathrm{UT}=1100$ and 1200 ( $\mathrm{LT}=1300-1400)$. The same phenomenon is also observed for impulsive chorus at $\mathrm{UT}=2100(\mathrm{LT}=2224)$. The times when this kind of phenomenon is detected are designated by asterisks at the top of Figure $5 a$.

\section{Results of Direction Findings}

Although many chorus emissions are recorded, as seen in Figures 3, 4, and 6, the number of emission events available for the direction finding is not so numerous, because the $300-\mathrm{Hz}$ SFA passband is stepped in frequency and it may happen not to be sitting at the right frequency at the right time. The grey-scale display of the spectrum of those chosen events is made so that we can distinguish chorus from other noises and estimate the $d f / d t$. We then examine the time stationarity of each chorus, by means of the algorithms of TREND and RUN [Bendat and Piersol, 1971], which is the most fundamental requirement of the present direction finding [Lefeuvre et al., 1982], and we make the spectral matrices at the relevant frequencies. The direction finding technique in section 2 is applied only to chorus events with sufficient $S / N$ ratio and with sufficient stationarity in time.
First, the results of direction finding of impulsive chorus are discussed. About 30 events, including both the upper and lower band frequencies, have been successfully analyzed. A study of eigenvalues of spectral matrices has shown that all of the impulsive chorus events satisfy the criterion of the singleplane wave model; in other words, the impulsive chorus is found to be composed of a single plane wàve. Figure 7 illustrates the distribution of wave normal directions of impulsive chorus observed from 0000 to $1200 \mathrm{UT}$ and from 2100 to 2200 UT. Figure $7 a$ refers to the $\theta$ distribution versus emission frequency normalized by $f_{H}\left(f / f_{H}\right)$, and the corresponding distribution of $\phi$ is plotted in Figure $7 b$. Since we use only the magnetic field components, there is an ambiguity of $180^{\circ}$ in the wave normal determination, and we have converted, in the figure, all the wave normal direction into those with $\theta$ less than $90^{\circ}$.

Now we look at the $\theta$ distribution in Figure 7a. Except one cross with its $\theta \sim 30^{\circ}$, all the crosses in the upper band are found to be located very close to the oblique resonance cone $\theta_{\text {res }}$ for the whistler mode in the cold plasma, indicated by a solid line in the figure, though, of course, the degree of closeness to $\theta_{\text {res }}$ is different from one cross to another. Furthermore, the four crosses with their $\theta$ close to $\theta_{\text {res }}$ in the lower band obviously correspond to emissions of the upper band extending down below $f_{H} / 2$. Hence we conclude that the upper band chorus is generated with its $\theta$ close to $\theta_{\text {res }}$

Another group of wave normals in the lower band is easily recognizable in Figure $7 a$. The $\theta$ values of this group are shown to lie in a range from $35^{\circ}$ to $50^{\circ}$; they are apparently far away from $\theta_{\text {res }}$ and have very different behaviors from those in the upper band. Thus the waves of this group are presumed to be excited by a mechanism different from that for the upper band. The $\phi$ values of the emissions belonging to this group are encircled in Figure $7 b$ for the sake of distinguishing them from those of the previous group.

The information on the $\phi$ distribution would be useful when the waves have propagated well away from the generation region, being focused into the meridian plane [Thorne, 1969]. The $\phi$ distribution above $f_{H} / 2$ in Figure $7 b$ may indicate that the $\phi$ are mainly in the quadrant $0^{\circ}$ to $90^{\circ}$, while the $\phi$ below 
$f_{H} / 2$ are distributed from $0^{\circ}$ to $180^{\circ}$, with some focusing around $\phi \simeq 90^{\circ}$. The distribution in azimuth near the source seems to depend strongly on the position of the satellite with respect to the source, but these $\phi$ distributions might suggest, at least, that the waves have not propagated over some distances, and they are generated close to the satellite or around the magnetic equator.

Impulsive chorus events analyzed in Figure 7 are already found to be of the one-direction model, and for this singleplane wave situation there exists an alternative method by Means [1972], on which the analyses by Burton and Holzer [1974] are based. Table 1 demonstrates a comparison of the results obtained by the present method with those obtained by the Means' method for randomly selected events. The two examples in the upper and lower bands correspond to the impulsive chorus, and the last ones correspond to the rising tone chorus identified as the one-direction model as shown in Figure 6. The table indicates that the discrepancy of the wave normal directions determined by both methods is of negligible order, the maximum difference in $\theta$ in the table being about $3^{\circ}$ and that in $\phi$ being about $5^{\circ}$. The differences for all other events lie within the above mentioned maximum deviations.

When we take into account the experimental fact that the $\theta$ values of the group mainly in the upper band, but extending sometimes down below $f_{H} / 2$, are very close to $\theta_{\text {res, }}$, it suggests that those waves are quasi-electrostatic. In order to have a further confirmation of this point, we have examined the ratio of $c|B| /|E|$, where $|B|$ and $|E|$ are the total magnetic and electric field intensities and $c$ is the light velocity. Figure 8 shows the distribution of the observed ratio, which indicates that there are two groups: in one the ratio is around 1.0, and in the other it is around 10. Even the high ratios around 10 are found to be still smaller than the corresponding refractive index of the electromagnetic whistler mode. The smaller ratio around 1.0 is clearly indicative of the quasi-electrostatic nature of the upper band chorus. Here we have to mention that although the observed waves are quasi-electrostatic, their magnetic field components are still observable, allowing us to use only the magnetic field components. Hashimoto and Kimura [1981] have estimated that $\left|E_{\perp}\right| /\left|E_{\|}\right|(\|$and $\perp$ denote parallel and perpendicular to the wave normal direction, respectively) decreases remarkably with increasing $\theta$ and the ratio becomes about $10^{-2}$ at $\theta$ close to $\theta_{\text {res, }}$ indicating that $E_{\|}$is very predominant. However, $|B| /|E|$ decreases to only one tenth of that at $\theta=0^{\circ}$ because of the increase in the refractive index. Hence the magnetic field components, as well as the electric field components, of those quasi-electrostatic mode waves are observable.

For comparison with the upper band chorus, the ratio $c|B| /|E|$ is determined for another group of chorus in the lower band with moderate $\theta$ angles. The ratio is found to lie in a range from 31 to 49 , which is very different from the results for the upper band chorus. It is apparent that this indicates that the lower band chorus is electromagnetic, although the observed ratio is greater by a factor of 1.5-2.0 than the corresponding refractive index of the whistler mode with moderate $\theta$.

Now we discuss the rising tone chorus. Figure 9 shows the summary of the wave normals determined for rising tone chorus. In the figure the wave normals indicated with arrows refer to the chorus events in Figure 6 which extend in frequency from lower to upper band without any change at $f_{H} / 2$. Other events analyzed correspond to chorus emissions for
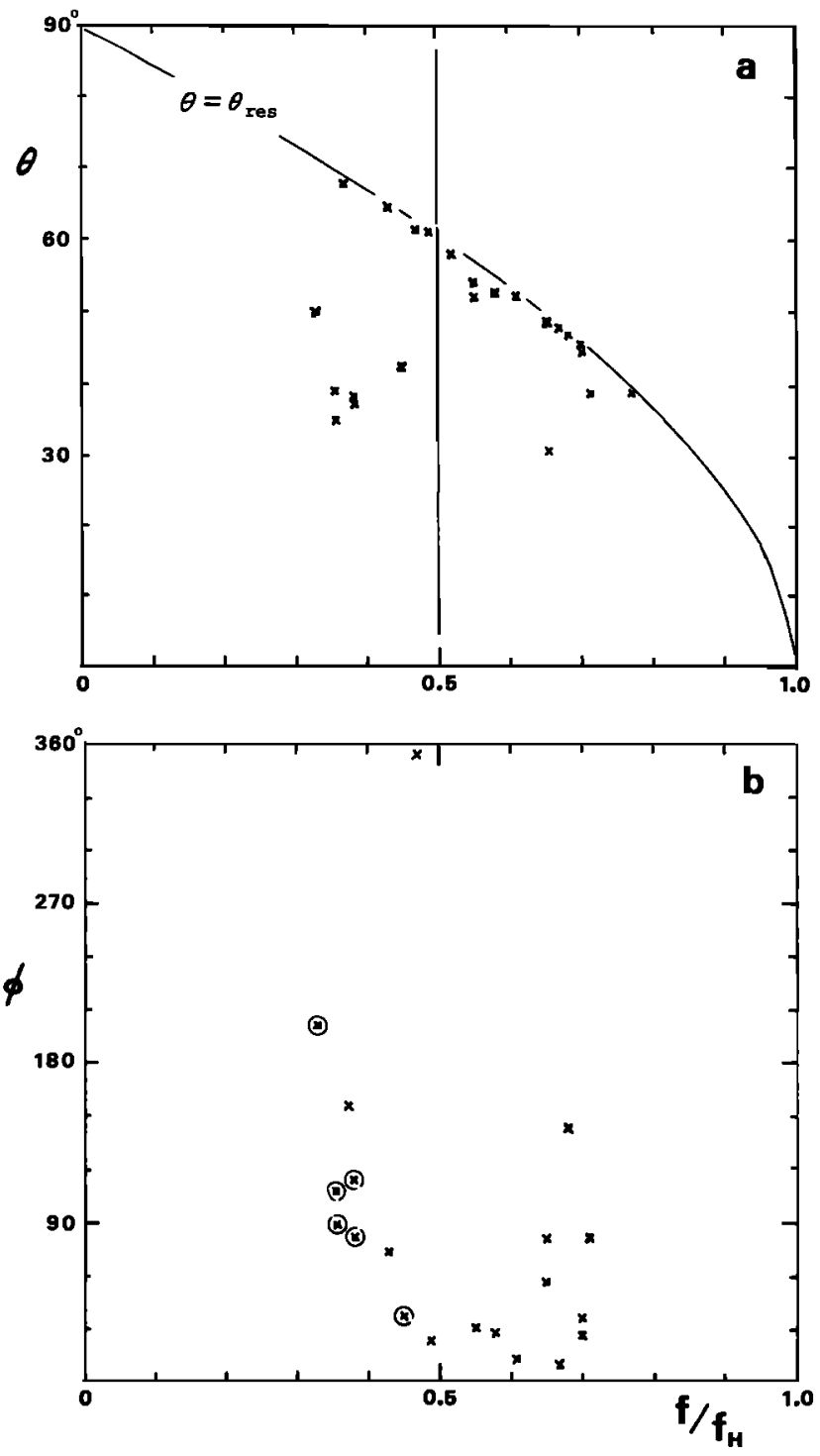

Fig. 7. Direction finding results for impulsive chorus; $(a)$ the angle $\theta$ between the wave normal and the earth's magnetic field and (b) the azimuthal angle $\phi$ are illustrated as a function of the normalized frequency, $f / f_{H}$. All of the observed chorus events are found to be composed of a single plane wave. The angles of the group of the waves with moderate $\theta$ angles are encircled to distinguish them from those with $\theta \leqslant \theta_{\text {res: }}$

which the upper band and lower band have no coherence, as in Figure 4; in other words, they occur independently of each other. Four events in the figure are identified as being composed of two plane waves, for which the energetic peak is expressed by a cross, as in the case of a single plane wave, and the secondary, less energetic peak by a small circle, linked with the corresponding cross by a dashed line. Other chorus events are found to be of the single-plane wave model, just as for the impulsive chorus in Figure 7. Figure $9 a$ shows the $\theta$ distribution versus the normalized emission frequency, and the corresponding $\phi$ distribution is given in Figure $9 b$.

First, we look at the wave normals of chorus without arrows. The $\phi$ dependence does not exhibit any particular concentration of any specific values. A similarity to the results for impulsive chorus is that there is one group, mainly in the upper band, extending sometimes down below $f_{H} / 2$, whose $\theta$ is 
TABLE 1. Comparison of the Direction Finding Results With the Present Method and With the Means' Method

\begin{tabular}{|c|c|c|c|c|}
\hline \multirow{2}{*}{$\begin{array}{l}\text { Time, } \\
\text { UT }\end{array}$} & \multirow{2}{*}{$\begin{array}{c}\text { Frequency, Hz, } \\
\text { Normalized Frequency }\end{array}$} & \multicolumn{2}{|c|}{$(\theta, \phi)$} & \multirow[b]{2}{*}{$\theta_{\text {res }}$} \\
\hline & & Present Method & Means' Method & \\
\hline $0001: 06$ & \multicolumn{2}{|c|}{ Upper Band Chorus } & $\left(54.3^{\circ}, 31.0^{\circ}\right)$ & $56.8^{\circ}$ \\
\hline $\begin{array}{l}\text { (impulsive) } \\
0901: 05 \\
\text { (impulsive) }\end{array}$ & $\begin{array}{c}0.55 \\
1371 \\
0.55\end{array}$ & $\left(52.2^{\circ}, 333.4^{\circ}\right)$ & $\left(50.8^{\circ}, 333.8^{\circ}\right)$ & $56.8^{\circ}$ \\
\hline $\begin{array}{l}1100: 43 \\
\text { (rising tone) }\end{array}$ & $\begin{array}{r}1620 \\
0.65\end{array}$ & $\left(21.8^{\circ}, 330.3^{\circ}\right)$ & $\left(21.7^{\circ}, 331.5^{\circ}\right)$ & $49.8^{\circ}$ \\
\hline \multicolumn{5}{|c|}{ Lower Band Chorus } \\
\hline (impulsive) & 0.355 & & & \\
\hline $\begin{array}{l}0901: 06 \\
\quad \text { (impulsive) }\end{array}$ & $\begin{array}{r}1214 \\
0.48\end{array}$ & $\left(39.5^{\circ}, 288.7^{\circ}\right)$ & $\left(36.8^{\circ}, 293.0^{\circ}\right)$ & $61.0^{\circ}$ \\
\hline $\begin{array}{l}1100: 45 \\
\text { (rising tone) }\end{array}$ & $\begin{array}{l}964 \\
0.38\end{array}$ & $\left(4.5^{\circ}, 287.4^{\circ}\right)$ & $\left(4.1^{\circ}, 292.7^{\circ}\right)$ & $67.3^{\circ}$ \\
\hline
\end{tabular}

again close to $\theta_{\text {res. }}$. However, there seems to be a difference from the case of impulsive chorus in that the closeness of the wave normals of this group to the oblique resonance cone is obviously much less distinct than in the case of impulsive chorus. Then, the lower band rising tone chorus is found to make moderate angles $\left(30^{\circ}-45^{\circ}\right)$ with $B_{0}$, these values being about $5^{\circ}$ smaller than those obtained for impulsive chorus in Figure $7 a$.

In Figure 9 we finally examine the wave normal behaviors of chorus with arrows as shown in Figure 6. In the lower frequency range below $f_{H} / 2$, the wave normals are found to make extremely small angles $\left(5^{\circ}-20^{\circ}\right)$ with $B_{0}$, and larger angles $\left(\sim 25^{\circ}\right.$ to $\left.\sim 35^{\circ}\right)$ are found for the upper band. There seems to be a tendency for the wave normal angle of chorus extending across $f_{H} / 2$ to increase with increasing frequency.

\section{Discussion of the Generation MechanisM OF CHORUS}

Although it is clear that dispersion (or the propagation effect) of chorus must take place [Koons et al., 1982], it certainly is not the major cause of the frequency-time characteristics detected in chorus tones [Burton and Holzer, 1974; Tsurutani and Smith, 1974], but a more probable explanation is nonlinear effects associated with the generation mechanism [Thorne, 1972]. The characteristic rising or falling tones of chorus elements have attracted much attention, and several mechanisms, such as particle phase bunching [Helliwell, 1967; Sudan and Ott, 1971] and wave potential well trapping [Nunn, 1974], have been proposed to explain these structures. The generation mechanism is also of interest.

We now discuss the generation mechanism of the more popular rising tone chorus. First, the rising tones which are continuous across $f_{H} / 2$ in Figure 6 are considered, and we have to note that the $d f / d t$ of the events analyzed is the smallest in the present paper, being closer to the value previously observed. This kind of chorus is also detected on board the GEOS 1 [Cornilleau-Wehrlin et al., 1978] and SCATHA [Koons, 1981] satellites. Because the emissions of this kind, though infrequent in occurrence, are likely to be different from all other rising tones to be discussed later, their wave normal properties are treated separately. Figure 9 has shown that the $\theta$ of these rising tones indicated by arrows increases from a very small angle (less than $20^{\circ}$ ) at $f / f_{H} \sim 0.4$ to $\sim 20^{\circ}-40^{\circ}$ in the upper band. The nature of the wave normals being nearly aligned with the magnetic field in the lower band is in good agreement with the result of Burton and Holzer [1974] for the rising tone chorus, and this gives further support to the generation in terms of loss cone instability. The chorus treated here can be supposed to belong essentially to the lower band chorus, with its uppermost frequency extending above $f_{H} / 2$, because of the larger temperature anisotropy [Kennel and Petschek, 1966]. When the two-frequency chorus was originally discovered by Tsurutani and Smith [1974], they proposed the damping mechanism for the missing band at $f_{H} / 2$ because chorus tones which cut across the $f_{H} / 2$ gap could clearly be observed. The GEOS 1 observation [CornilleauWehrlin et al., 1978] has indicated that the rising tone chorus emissions we are now discussing sometimes exhibit an extinct, but weak absorption band at $\int_{H} / 2$. These obvious observations imply that the generation mechanism for the upper band of these emissions is the same as for the lower band; in other words, the upper band is only an extended part of the lower

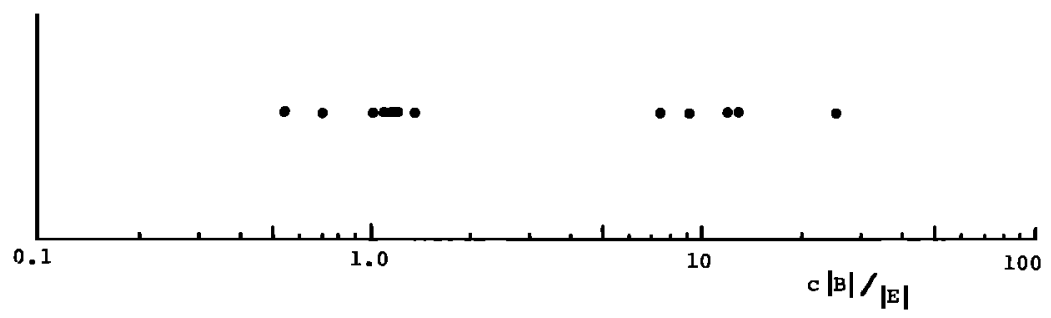

Fig. 8. Distribution of the observed ratio $c|B| /|E|$ of the chorus with $\theta \leqslant \theta_{\text {res: }}$ 
band, presumably originating from a loss cone instability, and also the missing gap at $f_{H} / 2$ is probably due to the damping effect, as proposed by Tsurutani and Smith [1974].

Next we discuss the wave normals of all other rising tone chorus emissions except those treated above. The direction finding analyses have demonstrated that the lower band chorus $\left(f=0.30-0.47 f_{H}\right)$ is generated with its wave normal making a moderate angle $\left(30^{\circ}-45^{\circ}\right)$ with the magnetic field, and these values are likely to be slightly larger than those obtained by Burton and Holzer [1974]; the possible reasons will be discussed later.

The impulsive chorus in the lower band has been found to be generated with their wave normals about $5^{\circ}$ larger than those of the rising tone chorus. There is a question whether different frequency-time structures are due to different wave normal directions. Cornilleau-Wehrlin et al. [1976] have made wave normal analyses for three different types of chorus (rising tone, falling tone, and burstlike) based on the Means' method for a small number of data from OGO 5. They have found that the falling tones take large wave normal angles $\left(50^{\circ}-60^{\circ}\right)$ while the wave normals of the rising tones are again very small, less than $20^{\circ}$.

Summarizing the above properties of wave normals of different types of chorus may lead us to suppose that different structures are associated with different wave normal directions. The values of $\theta$ of the rising tones with $d f / d t$ as normally observed have shown small angles such as $5^{\circ}-20^{\circ}$ in the present paper and less than $20^{\circ}$ in the work by Burton and Holzer [1974]. However, the rising tones with generally larger $d f / d t$ as studied in Figure 9 have indicated more oblique wave normals. The impulsive chorus seems to take larger $\theta$ values than the rising tones, and finally, the falling tones take much larger $\theta$ angles.

Since the wave normals of rising tones treated in the present paper (Figure 9) are slightly larger than those in the work by Burton and Holzer [1974], their nature has been considered here on the basis of the above characteristics of varying wave normals with varying different spectrograms. As is shown in section 3 , the $d f / d t$ of the rising tones in the present paper is found to be larger than that normally observed, and so it is possible that we would expect larger wave normals than the $\theta$ values obtained by Burton and Holzer. Another possibility might be related to the differences in electron distribution and in emission frequency. Kennel [1966] has shown that the angle of the unstable cone is larger, the harder the electron energy spectrum is, and also that for a fixed electron energy spectrum it is smaller, the higher the emission frequency is. It may be that the harder electron energy spectrum would have occurred in the present case.

The occurrence of different types of chorus might be related to the local time. Tsurutani and Smith [1974] have shown that flat and falling tones are detected primarily in the nightside magnetosphere at large $L$, and flat tones are rarely observed on the dayside. The present paper has shown that impulsive, burstlike chorus tends to be detected from postmidnight to afternoon, and rising tones appear only around noon at $L=6.6$. It would be interesting to investigate, in detail, the relationship of the frequency-time characteristic with $L T$ and $L$ value and the associated difference of wave normal directions with different structures.

We now discuss the generation mechanism of the upper band chorus. The present paper has clearly shown that the impulsive chorus in the upper band seems to be quasielectrostatic, making an angle very close to the oblique reso-
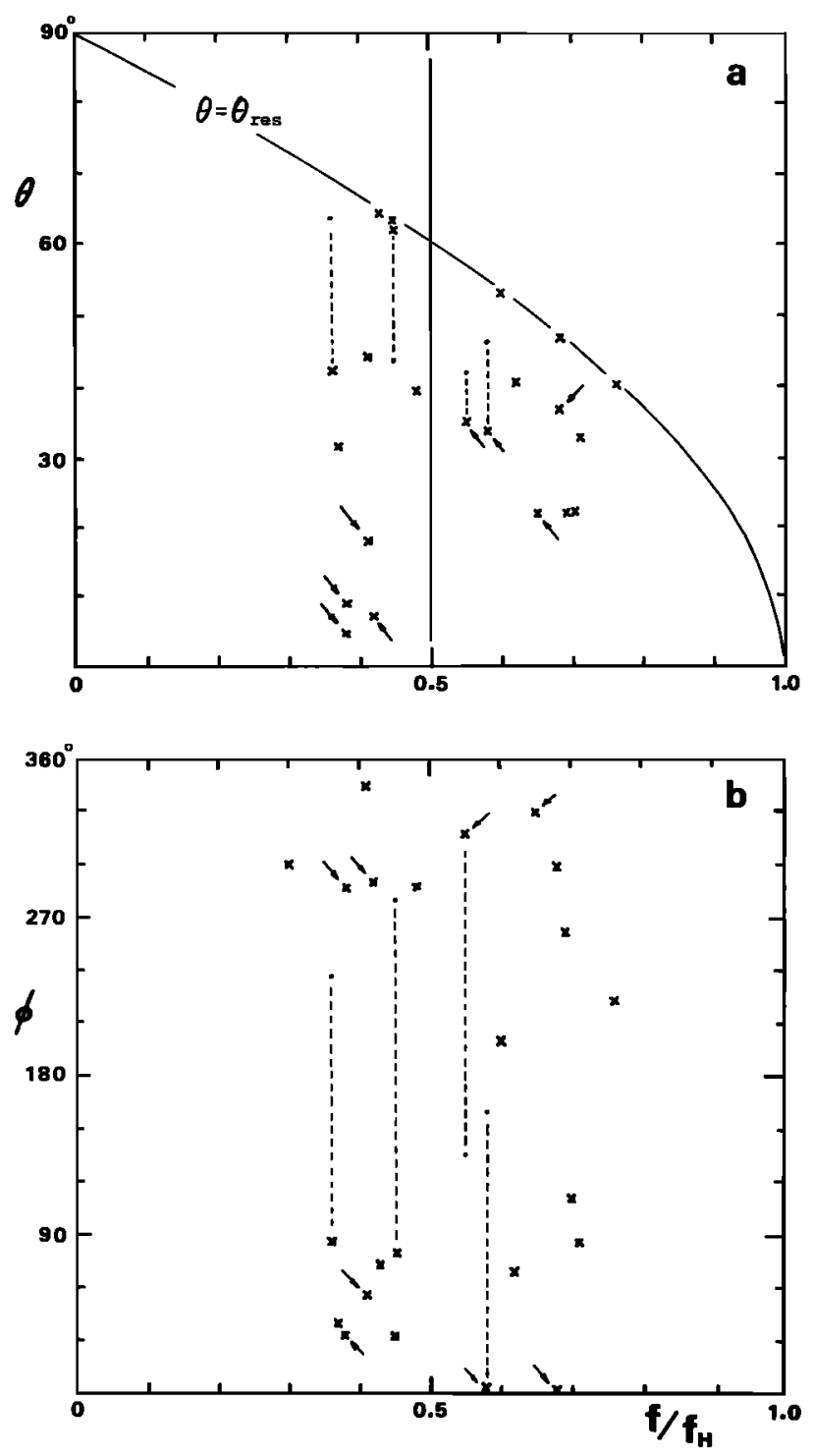

Fig. 9. Corresponding direction finding results for rising tone chorus for (a) the $\theta$ distribution and $(b)$ the $\phi$ angles. Many of the observed waves consist of a single plane wave, but some waves are of the two-plane wave case for which a cross indicates the energetic peak and the secondary one is represented by a small circle linked with the corresponding cross. The arrival directions indicated by arrows refer to the emissions in Figure 6.

nance cone. Maeda [1975], Maeda et al. [1976], and Anderson and Maeda [1977] have all suggested an electromagnetic instability due to an anisotropic electron velocity distribution, but such an electromagnetic instability is likely to be inapplicable to the upper band, which is quasi-electrostatic. Then, our direction finding studies appear to rule out the possibility suggested by Curtis [1978] that $f>f_{H} / 2$ waves are propagating in the ordinary mode. This is because the emission frequency is always below $f_{H}$, which is much smaller than $f_{p}$ (see Figure 2) and so the wave is whistler mode. An electrostatic instability has been postulated by Hashimoto and Kimura [1981] in order to attempt to explain $f>f_{H} / 2$ hiss. Since their mechanism is also applicable to chorus, it is interesting to compare their theoretical implications with our present experimental results. Their proposed model is a kind of Harris type electrostatic instability in a bi-Maxwellian plasma with temperature anisotropy, for which the quasi-electrostatic whistler mode with 
short wavelengths and large wave normal angles is unstable in a frequency range from $f_{H} / 2$ to an upper cutoff frequency dependent on the anisotropy factor. These theoretical predictions seem to be in agreement with the experimental results found in the present paper. Hashimoto and Kimura's emissions are predicted to propagate at an angle slightly larger than the oblique resonance cone angle on the assumption of the cold plasma theory; this seems to be in partial contradiction to our results of $\theta \leqslant \theta_{\text {res }}$ in Figure 7. This discrepancy is related to the assumption of the cold plasma describing the propagation of whister mode waves in the direction finding technique in section 2 . The cold plasma theory predicts that the whistler mode is allowed to propagate at an angle less than $\theta_{\text {res: }}$ When $\theta$ approaches $\theta_{\text {res }}$, the temperature effect should be included in the refractive index, which makes whistler mode propagation possible even for $\theta>\theta_{\text {reg}}$, as shown by Hashimoto and Kimura [1981]. Even if we take into account the temperature effect in the whistler mode refractive index in estimating the wave normal directions, it is safe to say that the final $\theta$ to be obtained would be around $\theta_{\text {res }}$, though we do not know whether the convergence in $\theta$ takes place above or below $\theta_{\text {res }}$. Furthermore, a small departure of the wave normal angles of some emissions from $\theta_{\text {res }}$ might be related to the propagation effect. Hashimoto and Kimura $[1973,1981]$ have suggested that their emissions should be highly electrostatic at the source region, but those excited by this electrostatic instability become less electrostatic (or more electromagnetic) with changing wave normals during the course of the propagation even in a small distance from the source region. This effect might be reflected in Figure 8. The wave normals of the rising tone chorus in the upper band in Figure $9 a$ are found to exhibit different behaviors from those for the impulsive chorus. Some of the wave normals depart considerably from $\theta_{\text {res, }}$ which seems to indicate that the transition from the electrostatic to the electromagnetic mode would be more noticeable for the rising tone upper band chorus. Further detailed theoretical investigation should be carried out until a definite conclusion is reached on the generation mechanism of the upper band chorus.

Acknowledgments. The authors would like to express their sincere thanks to P. Robert of CRPE (Issy les Moulineaux) for supplying the electron gyrofrequency data, and J. Etcheto and B. Higel of CRPE (Issy les Moulineaux) and P. Decreau of LPCE (Orléans) for supplying the plasma frequency measurements from the S-301 and S-304 experiments, respectively. Thanks are also due to CNES (Toulouse) for making the survey mode microfiches, some of which have been reproduced in the present paper. Useful discussions with J. Ohtsu and Y. Tanaka of Nagoya University, I. Kimura of Kyoto University, $\mathbf{H}$. Kikuchi of Nihon University, L. R. O. Storey and C. Beghin of LPCE, and B. T. Tsurutani of Jet Propulsion Laboratory are appreciated. The present work is partly supported by the grant-in-aid for scientific research (57580008) from Ministry of Education, Japan, and by the International Joint Project of Japan Society of Promotion of Science. Finally we are grateful to the referees for their useful criticism.

The Editor thanks C. C. Harvey and another referee for their assistance in evaluating this paper.

\section{REFERENCES}

Anderson, R. R., and K. Maeda, VLF emissions associated with enhanced magnetospheric electrons, J. Geophys. Res., 82, 135, 1977.

Bendat, J. S., and A. G. Piersol, Random Data: Analysis and Measurement Procedures, Wiley-Interscience, New York, 1971.

Buchalet, L. J., and F. Lefeuvre, One and two direction models for VLF electromagnetic waves observed on-board GEOS 1, J. Geophys. Res., 86, 2377, 1981.

Burtis, W. J., and R. A. Helliwell, Banded chorus: A new type of VLF radiation observed in the magnetosphere by Ogo 1 and Ogo $3, J$. Geophys. Res., 74, 3002, 1969.

Burtis, W. J., and R. A. Helliwell, Magnetospheric chorus: Occurrence patterns and normalized frquency, Planet. Space Sci., 24, 1007. 1976.

Burton, R. K., and R. E. Holzer, The origin and propagation of chorus in the outer magnetosphere, J. Geophys. Res., 79, 1014, 1974.

Cornilleau-Wehrlin, N., J. Etcheto, and R. K. Burton, Detailed analysis of magnetospheric chorus: Preliminary results, J. Atmos. Terr., Phys., 38, 1201, 1976.

Cornilleau-Wehrlin, N., R. Gendrin, F. Lefeuvre, M. Parrot, R. Grard, D. Jones, A. Bahnsen, E. Ungstrup, and W. Gibbons, VLF electromagnetic waves observed onboard GEOS-1, Space Sci. Rev., $22,371,1978$.

Curtis, S. A., A theory on chorus generation by energetic electrons during substorms, J. Geophys. Res., 83, 3841, 1978.

Decreau, P. M. E., C. Beghin, and M. Parrot, Electron density and temperature, as measured by the mutual impedance experiment on board GEOS-1, Space Sci. Rev., 22, 581, 1978.

Dunckel, N., and R. A. Helliwell, Whistler-mode emissions on the OGO satellite, J. Geophys. Res., 74, 6371, 1969.

Etcheto, J., and J. J. Bloch, Plasma density measurements from the GEOS-1 relaxation sounder, Space Sci. Rev., 22, 597, 1978.

Gibbons, W., and I. A. Ward, A preliminary analysis of the ELF/VLF signals received on-board GEOS 2 as detected by the filter-bank, Adv. Space Res., 1, 361, 1981.

Hashimoto, K., and I. Kimura, Oblique whistler mode propagation in a hot plasma, Rep. Ionos. Space Res. Jpn., 27, 73, 1973.

Hashimoto, K., and I. Kimura, A generation mechanism of narrow band hiss emissions above one half the gyrofrequency in the outer magnetosphere, J. Geophys. Res., 86, 11,148, 1981.

Hayakawa, M., K. Bullough, and T. R. Kaiser, The properties of storm-time magnetospheric VLF emissions as deduced from the Ariel-3 satellite and ground-based observations, Planet. Space Sci., 25, 353, 1977.

Helliwell, R. A., A theory of discrete VLF emissions from the magnetosphere, J. Geophys. Res., 72, 4773, 1967.

Isenberg, P. A., H. C. Koons, and J. F. Fennel, Simultaneous observations of energetic electrons and dawnside chorus in geosynchronous orbit, J. Geophys. Res., 87, 1495, 1982.

Jones, D., Introduction to the S-300 wave experiments onboard GEOS, Space Sci. Rev., 22, 327, 1978.

Kennel, C. F., Low-frequency whistler mode, Phys. Fluids, 9, 2190, 1966.

Kennel, C. F., and H. E. Petschek, Limit of stably trapped particle fluxes, J. Geophys. Res., 71, 1, 1966.

Koons, H. C., The role of hiss in magnetospheric chorus emissions, $J$. Geophys. Res., 86, 6745, 1981.

Koons, H. C., C. Hetlinger, and B. C. Edgar, A mechanism to explain the shapes of chorus emissions, Rep. SSL-823(7954), Space Sci. Lab., Aerospace Corp., Los Angeles, Calif., 1982.

Lefeuvre, F., T. Neubert, and M. Parrot, Wave normal direction and wave distribution functions for ground-based transmitter signals observed on GEOS 1, J. Geophys. Res., 87, 6203, 1982.

Maeda, K., Cyclotron side-band emissions from ring current electrons, Planet. Space Sci., 24, 341, 1975.

Maeda, K., P. H. Smith, and R. R. Anderson, VLF emissions from ring current electrons, Nature, 262(5572), 37, 1976.

Means, J. D., The use of the three-dimensional co-variance matrix in analyzing the properties of plane waves, J. Geophys. Res., 77, 5551, 1972.

Nunn, D., A self-consistent theory of triggered VLF emissions, Planet. Space Sci., 22, 349, 1974.

Oliven, M. N., and D. A. Gurnett, Microburst phenomena, 3, An association between microbursts and VLF chorus, J. Geophys. Res., $73,2355,1968$.

Russell, C. T., and R. E. Holzer, AC magnetic fields, in Particles and Fields in the Magnetosphere, edited by B. M. McCormac, p. 195, D. Reidel, Hingham, Mass., 1970.

Russell, C. T., R. E. Holzer, and E. J. Smith, Ogo 3 observations of ELF noise in the magnetosphere, 1, Spatial extent and frequency of occurrence, J. Geophys. Res., 74, 755, 1969.

S-300 experimenters, Measurements of electric and magnetic wave fields and of cold plasma parameters onboard GEOS-1, Preliminary results, Planet. Space Sci., 27, 317, 1979.

Sudan, R. N., and E. Ott, Theory of triggered VLF emissions, $J$. Geophys. Res., 76, 4463, 1971.

Taylor, W. W. L., and D. A. Gurnett, Morphology of VLF emissions 
observed with the Injun 3 satellite, J. Geophys. Res., 73, 5615, 1968.

Thorne, R. M., Whistler mode propagation off the geomagnetic meridian plane, internal report, Dep. of Meteorol., Univ. of Calif., Los Angeles, 1969.

Thorne, R. M., The importance of wave-particle interactions in the magnetosphere, in Critical Problems of Magnetospheric Physics, edited by E. R. Dyer, p. 211, National Academy of Sciences, Washington, D. C., 1972.

Thorne, R. M., E. J. Smith, K. J. Fiske, and S. R. Church, Intensity variations of ELF hiss and chorus during isolated substorms, Geophys. Res. Lett., 1, 193, 1974.

Thorne, R. M., S. R. Church, W. J. Malloy, and B. T. Tsurutani, The local time variations of ELF emissions during periods of substorm activity, J. Geophys. Res., 82, 1585, 1977.

Tsurutani, B. T., and E. J. Smith, Postmidnight chorus: A substorm phenomenon, J. Geophys. Res., 79, 118, 1974.

Tsurutani, B. T., and E. J. Smith, Two types of magnetospheric ELF chorus and their substorm dependences, J. Geophys. Res., 82, 5112, 1977.
Tsurutani, B. T., E. J. Smith, H. I. West, Jr., and R. M. Buck, Chorus, energetic electrons and magnetospheric substorms, in Wave Instabilities in Space Plasma, edited by P. J. Palmadesso and K. Papadopoulos, p. 55, D. Reidel, Hingham, Mass., 1979.

Wrenn, G., Geos 2 in space collision?, Nature, 274, 631, 1978.

M. Hayakaya, Research Institute of Atmospherics, Nagoya University, 13 Honohara 3-chome, Toyokawa 442, Japan.

L. Lefeuvre and M. Parrot, Laboratoire de Physique et Chimie de l'Environnement, Centre National de la Recherche Scientifique, 3A, avenue de la Recherche Scientifique, 45045 Orléans Cedex, France.

Y. Yamanaka, Radio Research Laboratories, Koganei, Tokyo 184, Japan.

(Received June 7, 1983;

revised December 28,1983

accepted January 11,1984 .) 